\title{
Embracing industry sponsored research to expand osteopathic medical student research experiences
}

https://doi.org/10.1515/jom-2021-0178

Received June 30, 2021; accepted August 17, 2021;

published online September 3, 2021

To the Editor:

Two recent articles published in the Journal of Osteopathic Medicine - an Editorial by Dr. Elizabeth A. Beverly [1] and a Letter to the Editor by OMS Steven J. Doyle [2] - addressed the importance of building and supporting a research culture in osteopathic medical schools and highlighted many of the current barriers reducing research output by osteopathic medical students compared to their allopathic colleagues. As pointed out by Dr. Beverly [1], research experiences and publications are proving increasingly important in the single match system as another facet of a complete graduate medical education (GME) residency application [3]. While we agree with OMS Doyle [2] on the importance of supporting physician scientist training programs to further the osteopathic research culture, we propose that it's time to embrace industry-sponsored research training experiences to help fill the gap in research training opportunities for osteopathic medical students.

During the COVID-19 pandemic, private corporations from the biotechnology and pharmaceutical industries worked alongside governmental and academic researchers at record speeds to address the global needs for diagnostic, therapeutic, and vaccine development [4]. These collaborations quickly produced high quality

*Corresponding author: Zachary P. Morehouse, OMS IV, MS, Michigan State University College of Osteopathic Medicine, 965 Wilson Road, East Lansing, MI 48824, USA; and Omni International, Inc., A PerkinElmer Company, Kennesaw, GA, USA,

E-mail: moreho17@msu.edu

Rodney J. Nash, PhD, Omni International, Inc., A PerkinElmer Company, Kennesaw, GA, USA; and Department of Biology, Georgia State University, Atlanta, GA, USA data that was used to constantly reevaluate and evolve the management of this pandemic at every level, from individual patients to global public health strategies. It is our hope that the synergistic relationship between academic and industry research will continue to flourish to address many of the other medical issues facing our world.

Herein, we propose that just as osteopathic medical schools have innovated their delivery of clinical medical education by sending medical students to community hospital training sites for their rotations, they can engage with the research community in the same way by supporting their students to obtain research internship opportunities within biotechnology and pharmaceutical industries. These experiences will provide quality training opportunities for future osteopathic physicians interested in gaining research training, as well as give exposure to how these industries (that they will undoubtedly interact with during their professional careers) conduct the research needed to bring new products to market to address clinical issues. While we are neither suggesting abandonment of the current physician scientist training model nor are we downplaying the importance of academic research experiences in medical education, we feel that this additional avenue for exposure to research may be another pathway to fostering a growing research culture in osteopathic medical education.

The described industry partnered research experiences have been successfully implemented in some masters and doctoral level graduate training programs, such as the graduate training programs at Georgia State University and the University of Georgia, which allowed both authors of this Letter to the Editor to participate in industry partnered research as a core component of their graduate training. These experiences, which have helped pave the way for collaboration between academic, clinical, and industry players in research projects, have fostered a mindset of collaboration for innovation that has proven critical in the response to COVID-19 and can 
continue with this focus on innovation to support the patients who need it most.

We firmly acknowledge that there are barriers to implementing these types of experiences into osteopathic medical education, such as negative public opinions surrounding the pharmaceutical and biotechnology industries, and difficulties in establishing protected research time in a very busy medical education schedule [5]. While difficult, implementing protected research time for osteopathic medical students is not an impossible ask given that such opportunities are offered by many osteopathic and allopathic colleges during both pre-clerkship and clerkship semesters. Through utilization of existing summer internship programs offered by industry affiliates, or local collaborations with clinical trial networks or regionally located companies, we feel that there is a path forward for these research opportunities that is worth investigating.

While it would be naive to expect these experiences to be without difficulties, open dialogue between medical colleges and industry affiliates on the differences in research cultures and expectations for involved students should provide a starting point to build these relationships. Through transparency from both sides on expectations and goals for involved students, we think there will be more common ground seen than many critics would propose in opposition to these programs. While industry does conduct research with the end goal of developing technology to bring to market, this is not done in a vacuum and students involved in these projects would gain the same skills in literature review, laboratory techniques, data set management, and manuscript preparation as they would in an academic research setting. Additionally, any industry scientist interested in bringing a student aboard to assist in their projects is likely just as willing and eager to provide mentorship and teaching to students as any academic researcher willing to bring on such students. Perhaps these partnerships would help bring the academic and industry sectors closer together on common ground to focus on addressing the needs of our patients.

Transparency of expectations from all parties and a student-focused mentality are the critical components needed to build the proposed research experience for osteopathic medical students. Frequent meetings between medical college leadership, involved students, and the industry partners with open dialogue regarding the status of the programs will be essential to allow these partnerships to flourish and ensure that students are benefitting. We do not deny the fact that these programs will likely have some growing pains, but through transparent and open communication we are hopeful that all involved parties would continue to adapt and grow them into robust research experiences for osteopathic medical students and strong partnerships benefiting both sides.

Involving osteopathic medical students in industry supported research roles will provide benefits to all involved. It will expand research training opportunities to the students without concerns of obtaining funding, while the sponsoring company will benefit from the assistance on the projects as well as help in identify key needs of research in that community. Improving the research culture in osteopathic medical education will require support from multiple directions, and while these conversations appear to be happening with increasing frequency, we feel the use of industry sponsored research experiences should be considered as another pathway to help foster the growing research culture for the osteopathic medical profession.

Research funding: None reported.

Author contributions: Both authors provided substantial contributions to conception and design, acquisition of data, or analysis and interpretation of data; both authors drafted the article or revised it critically for important intellectual content; both authors gave final approval of the version of the article to be published; and both authors agree to be accountable for all aspects of the work in ensuring that questions related to the accuracy or integrity of any part of the work are appropriately investigated and resolved.

Competing interests: Mr. Morehouse is an employed consultant with Omni International, Inc. and Dr. Nash is a full-time employee of Omni International, Inc.

\section{References}

1. Beverly EA. Building an osteopathic research culture. J Osteopath Med 2021;121:333-5.

2. Doyle SJ. The importance of osteopathic physician scientist training programs. J Osteopath Med 2021;121:599.

3. National Resident Matching Program. Data Release and Research Committee: results of the 2018 NRMP Program Director Survey. Washington, DC: National Resident Matching Program; 2018.

4. Slaoui M, Hepburn M. Developing safe and effective covid vaccines - operation warp speed's strategy and approach. $\mathrm{N}$ Engl J Med 2020;383:1701-3.

5. Olsen AK, Whalen MD. Public perceptions of the pharmaceutical industry and drug safety: implications for the pharmacovigilance professional and the culture of safety. Drug Saf 2009;32: 805-10. 